\title{
Dynamics of a stainless steel turning process by statistical and recurrence analyses
}

\author{
Grzegorz Litak • Rafał Rusinek
}

Received: 6 June 2010 / Accepted: 21 November 2011 / Published online: 3 January 2012

(C) The Author(s) 2011. This article is published with open access at Springerlink.com

\begin{abstract}
This paper explores the cutting force oscillations. Forces have been measured during the stainless steel turning. We provide the results of standard statistical analysis of the corresponding time series together with their recurrence properties. We claim that the system, which initially exhibits regular vibrations, is unstable to chaotic oscillation for some fairly larger cutting depths. This characteristic transition in the cutting dynamics can be monitored by recurrences and could have the important implications to design a new control procedure.
\end{abstract}

Keywords Cutting process - Turning process . Nonlinear vibrations · Mechanics of machines . Recurrence plots

\section{Introduction}

The leading manufacturing technologies to obtain designed surface use the turning process. Unfortunately, in certain working conditions this process is unstable to chatter vibrations. They destroy the process precision and finally lower the product quality. Besides the process precision and the final product quality the large amplitude of chatter vibrations can brake the tool or damage the machine tool itself.

G. Litak $(\varangle) \cdot$ R. Rusinek

Department of Applied Mechanics, Lublin University of Technology, Nadbystrzycka 36, 20-618 Lublin, Poland e-mail: g.litak@pollub.pl
Initially, the appearance of unexpected chatter was studied by Taylor [1]. The first step toward the successful explanation of the chatter vibrations was made by Arnold [2]. He analyzed the self-sustained mechanism of vibrations. The subsequent studies show the importance of regenerative effects [3-6], and structural dynamics [7-9]. Finally, the role of the dry friction and the stick-and-slip motion [10-12] was indicated and studied extensively. Recently, apart from the widely studied conditions of regular chatter vibrations, noise like chaotic oscillations caused by various system nonlinearities were predicted and detected [13-25].

Over the past decade, the fast development in cutting technology gave way to a reliable high-speed cutting procedure. Consequently, elimination and stabilization of the associated chatter oscillations have become a high interest in science and technology [26]. The plausible adaptive control concept, based on relatively short time series, has been studied to gain deeper understanding. In this paper we investigate dynamics of a turning process (Fig. 1) by statistical and recurrence analysis. Applying these methods to the experimental time series of cutting forces in the stainless steel turning process enables us to distinguish different types of the system response. Namely, by changing the single system parameter, cutting depth $h_{0}$, we observed qualitative change of the system response. The knowledge about dynamics of this process can be used to optimize the process to obtain surfaces of the better quality. 


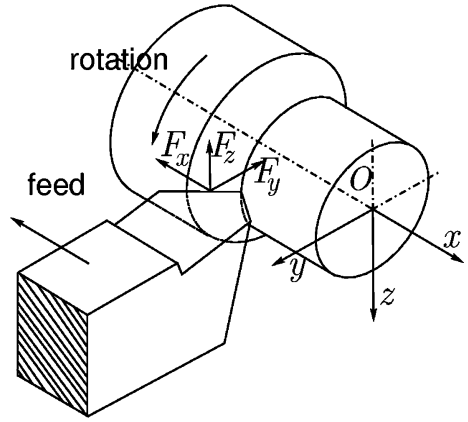

Fig. 1 Physical model of a regenerative turning process. The three orthogonal measured force components: feed $F_{x}$, radial $F_{y}$, and tangential $F_{z}$

The paper is organized as follows: We provide the experimental procedure, the results of the cutting force components in the time domain, and the basic statistics in Sect. 2. Section 3 is devoted to the recurrence plots analysis. The paper ends with Sect. 4 -conclusions.

\section{Experimental procedure and results}

Our turning experiment has been conducted using the workpiece circular shaft of stainless steel (EZ6NCT25) with the diameter of $22 \mathrm{~mm}$ and the tool cutting edge angle of 45 degrees. The shaft angular velocity was fixed to about $780 \mathrm{rpm}$ while the corresponding feeding ratio was $0.25 \mathrm{~mm} / \mathrm{rev}$. The experiment have been repeated for three different cutting depths $h_{0}$. Note that the actual cutting depth was not constant, but $h_{0}$ can be considered as the average cutting depth.

The obtained results consists of the three orthogonal force components (feed $F_{x}$, radial $F_{y}$, and tangential $F_{z}$ components, respectively-Fig. 1). They have been measured with the sampling frequency of $2 \mathrm{kHz}$. The measured data have been presented in Fig. 2 as the corresponding time series. To show the dominating trend with changing $h_{0}$ the 3D force diagram was plotted in Fig. 3. Additionally, in Table 1 we specified the statistical parameters (standard deviation- $S D$, skewness- $S K$, kurtosis- $K U$, and root mean square-RMS) related to the corresponding force components.

Note that in this note we show the results of this method limiting to the fairly small time interval composed of 1500 measurement points. The aim of our consideration is to find and follow the force evolution patterns by changing the cutting depth $h_{0}$.
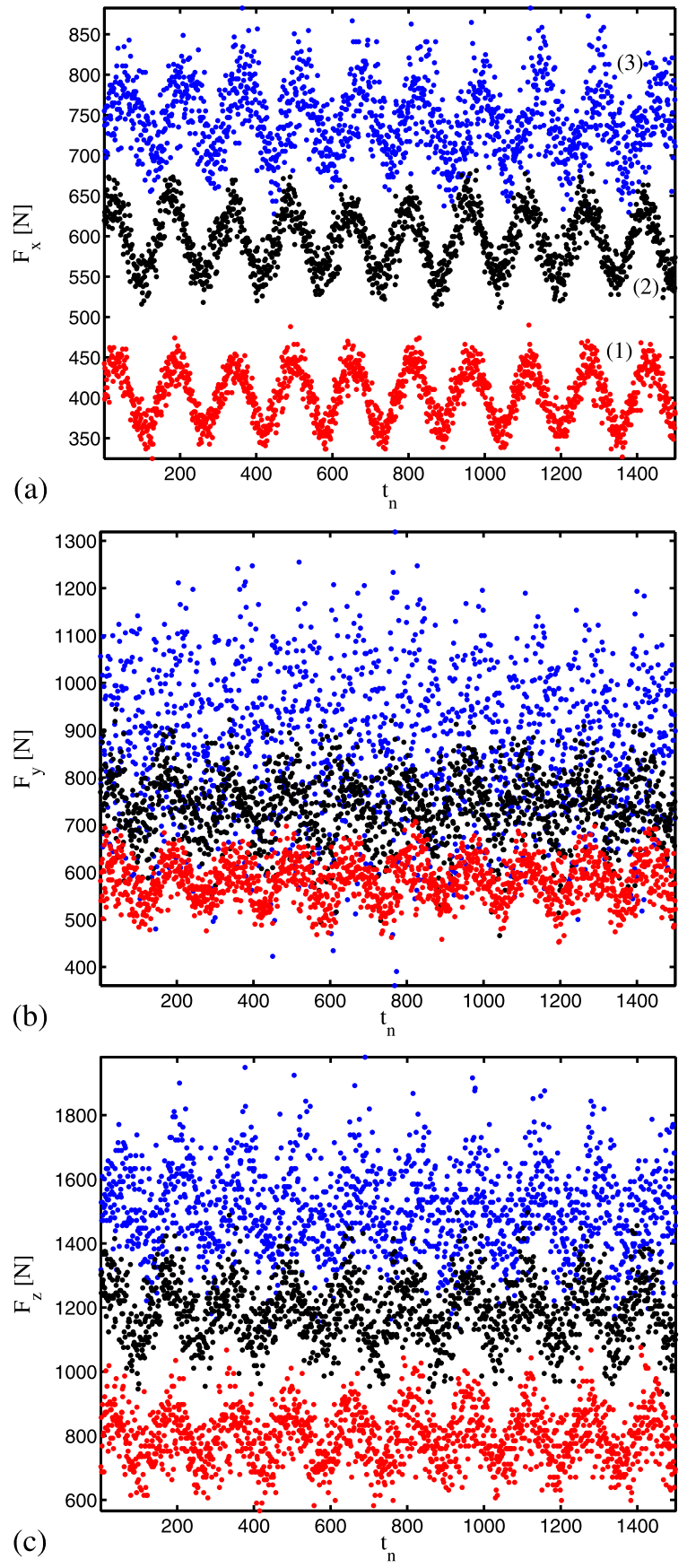

Fig. 2 (Color online) Time series of the cutting force components at various cutting depths $h_{0}=1.00,1.75$, and $2.30 \mathrm{~mm}$, for (1)—red, (2)—black, and (3)—blue respectively. The sampling frequency was $2 \mathrm{kHz}$, while the number of considered measurement points $N=1500$

Figure 3 illustrates the distribution of measured points for the corresponding cutting depth $\left(h_{0}=\right.$ $1.00,1.75$, and $2.30 \mathrm{~mm}$, respectively). Note that all 


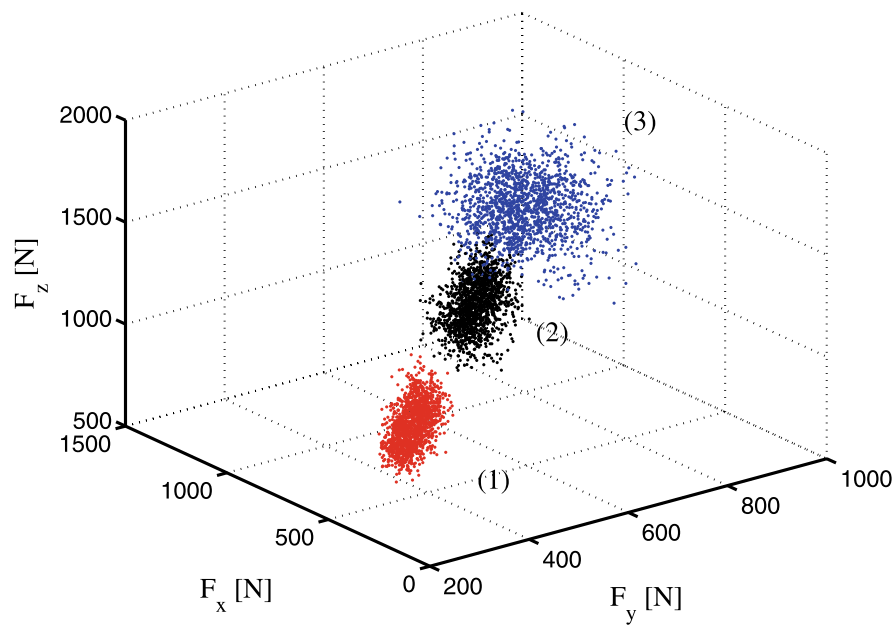

Fig. 3 The 3D force diagram. Points (1), (2), and (3) denote the cutting force measurement points for the cutting depth $h_{0}=1.00$, 1.75 , and $2.30 \mathrm{~mm}$, respectively. The sampling frequency was $2 \mathrm{kHz}$, while the number of considered measurement points $N=1500$

Table 1 Summary of statistical analysis

\begin{tabular}{llllll}
\hline $\begin{array}{l}\text { Cutting depth } \\
h_{0}[\mathrm{~mm}]\end{array}$ & $\begin{array}{l}\text { Average } M V_{i} \\
F_{i}, i=x, y, z[N]\end{array}$ & $\begin{array}{l}\text { Standard deviation } S D_{i} \\
\sigma_{F_{i}}, i=x, y, z[N]\end{array}$ & $\begin{array}{l}\text { Kurtosis } K U_{i} \\
i=x, y, z\end{array}$ & $\begin{array}{l}\text { Skewness } S K_{i} \\
i=x, y, z\end{array}$ & $\begin{array}{l}R M S_{i} \\
i=x, y, z\end{array}$ \\
\hline 1.00 & $403.5,581.2,794.9$ & $32.6,45.0,86.5$ & $2.016,2.591,2.821$ & $-0.041,0.013,0.240$ & $404.8,582.9,799.6$ \\
1.75 & $594.6,740.9,1189.1$ & $36.2,68.8,97.3$ & $2.102,3.400,2.847$ & $0.037,0.253,0.089$ & $595.7,744.1,1193.1$ \\
2.30 & $743.5,869.6,1501.8$ & $44.9,151.1,131.9$ & $2.628,2.661,3.055$ & $0.157,0.153,0.204$ & $744.9,882.6,1507.5$ \\
\hline
\end{tabular}

three averages of the force components are increasing monotonically with increasing $h_{0}$ (Figs. 2 and 3 ) as expected. Furthermore, the fluctuation range of cutting force components is also increasing. The summary of their statistical properties (Table 1) confirms this trend in terms of the standard deviations $S D_{i}$ (where $i=x, y, z)$. Interestingly, for the smaller $h_{0}(1.00$ and $1.75 \mathrm{~mm}$ ) the dominant fluctuations are $z$-component (see the $D S_{i}$ in Table 1) while for the largest $h_{0}$ $\left(h_{0}=2.30 \mathrm{~mm}\right) y$-force component. In other words the $y$-component fluctuations are developing faster than others.

Skewness $S K_{i}$ is relatively small for all cases indicating the left-to-right mirror symmetry of distribution. Finally, kurtosis values $K U_{i}$ are relatively close to that of the Gaussian distribution 3.0 for $i=z$ force components. On the other hand for the feed force component $(i=x)$ we observe the typical leptokurtic density $\left(K U_{x}<3\right)$, characterized with more peaky distribution. The last case of the radial force component $(i=y)$ is the most interesting. For instance, by the cutting depth $h_{0}=1.00$ and $2.30 \mathrm{~mm}$ the distribution is leptokurtic ( $K U_{y}=2.591$ and 2.661, respectively) while for $h_{0}=1.75 \mathrm{~mm}$ we observe the platykurtic $\left(K U_{y}>3\right.$, more flat than the Gaussian) distribution with kurtosis $K U_{y}$ reaching 3.40. This is the largest discrepancy from the monotonic tendencies and may signal intermittency at $h_{0}=1.75 \mathrm{~mm}$. The effect of the fluctuations increase can be also measured by the $R M S_{i}$ (Table 1). One can see that $M S R_{i}-M V_{i}$ (for $i=x, y, z)$ is the largest for $y$ component of force in the $h_{0}=2.30 \mathrm{~mm}$. Note also, the nonlinear nonmonotonous dependence of $h_{0} M S R_{y}-M V_{y}=1.7$, 3.3, 13.0, while for $x$ and $z$ components this difference is only slightly changing with $h_{0}$.

More extensive analyze is needed to tell more about this transition and the difference between dynamical response of the $h_{0}=1.00$ and 2.30 oscillations. For that reason we performed the covariance matrix analysis.

$$
\begin{aligned}
\operatorname{COV}\left(h_{0}\right)_{i j}= & \frac{1}{N} \sum_{k}\left(F_{i}\left(h_{0}, k\right)-\bar{F}_{i}\left(h_{0}\right)\right) \\
& \times\left(F_{j}\left(h_{0}, k\right)-\bar{F}_{j}\left(h_{0}\right)\right),
\end{aligned}
$$


where the $i, j=x, y, z$ denote the force components, and $k=1, \ldots, N$ is the time series sampling index. After simple calculations we obtain covariance matrices $\mathbf{C O V}^{1}, \mathbf{C O V}^{2}, \mathbf{C O V}^{3}$ for $h_{0}=1.00,1.75$, $2.30 \mathrm{~mm}$, respectively.

$$
\begin{aligned}
& \mathbf{C O V}^{1}=\left[\begin{array}{ccc}
1064 & 791 & 1367 \\
791 & 2029 & 1180 \\
1367 & 1180 & 7481
\end{array}\right] \mathrm{N}^{2}, \\
& \mathbf{C O V}^{2}=\left[\begin{array}{ccc}
1310 & 1067 & 1737 \\
1067 & 4735 & 1219 \\
1737 & 1219 & 9460
\end{array}\right] \mathrm{N}^{2}, \\
& \mathbf{C O V}^{3}=\left[\begin{array}{ccc}
2018 & 576 & 1297 \\
576 & 22838 & -2989 \\
1297 & -2989 & 17398
\end{array}\right] \mathrm{N}^{2} .
\end{aligned}
$$

To illustrate the differences in the matrix elements we plotted them in 3D diagrams (Figs. 4(a)-(c)). The diagonal values of the matrices $\mathbf{C O V}^{i}$ (equation (2)) are corresponding variances $V A R_{j}=C O V_{j j}^{i}=\left(S D_{j}\right)^{2}$ (where $S D_{j}, j=x, y, z$ are specified in Table 1). The off-diagonal values show the coupling between the corresponding force components. Figure 4 shows that most of couplings are fairly small. Note that $y$ force component is very weakly coupled with $y$ and $z$. The exception is $x$ component which could be driven by $z$ by relatively large coupling. Interestingly, the coupling phase between $y$ and $z$ components is changing with $h_{0}$. For $h_{0}=1.00$ and $1.75 \mathrm{~mm}$ it is positive while for $h_{0}=2.30 \mathrm{~mm}$ it is changing to negative.

In the next section we apply the recurrence analysis by recurrence plots quantified by recurrence rate $R R$ and other parameters related to the diagonal and vertical lines distributions. To achieve that we reconstruct the embedding space using single and multivariant signals of the measured force components at given sampling events and the corresponding time delayed quantities.

\section{Recurrence analysis}

To perform recurrence plot analysis we have to construct a phase space using the measured 3 dimensional (multi-variant) signal $\left[F_{x}(k), F_{y}(k), F_{z}(k)\right]$, where $k$ is the a natural number indexing the sampling points $k=1, \ldots, N$. Following Takens theorem [27], the state of the system can be reconstructed through the discrete time delay vectors. Assuming the total embedding dimension as $m=6$ we include the time delay coordinates:

$$
\begin{aligned}
\mathbf{F}(k)= & {\left[F_{x}(k), F_{y}(k), F_{z}(k), F_{x}(k-\Delta k),\right.} \\
& \left.F_{y}(k-\Delta k), F_{z}(k-\Delta k)\right] .
\end{aligned}
$$

Note that $\Delta k$ is the corresponding time delay in sampling interval units (the sampling frequency was $2 \mathrm{kHz}$ ).

It should be noted that the standard methods to determine the $\Delta k$ is the Average Mutual Information (AMI) or the autocorrelation function [28] but here we decided to use a much simpler approach [29]. We noted the dominating period in the time series $T \approx 190$ and the $\Delta i$ as one quarter of that. This approximate procedure has been tested many times for the system which show the clear periodicity [30,31]. Thus, in our case, $\Delta i=45$.

After reconstruction of the phase space one can calculate the Euclidean distance matrix $\mathbf{R}$ using the independent time delayed coordinates:

$\mathbf{R}_{i j}=\Theta(\epsilon-\|\mathbf{F}(i)-\mathbf{F}(j)\|), \quad$ for $|i-j| \geq w$,

where $\epsilon$ is a threshold value which has a meaning of the tolerance of recurrence and $\Theta($.$) is the Heaviside$ step function, $w$ is the Theiler window used to exclude identical and neighboring points from the analysis. In our case we adopted $w=1$. The distance matrix consists of zeros and ones that correspond to the state of the system in the time instant $j$ with respect to the time instant $i$ ( 1 -recurrence, 0 -no recurrence). In Figs. 5(a)-(c) we present the graphical representation of the distance matrix which is called a recurrence plot (RP) [33]. The threshold value has been chosen universally of all the cases by $\epsilon=\overline{S D}$. Note that $\epsilon$ is expressed in terms of the standard deviations product of $x, y, z$ force components:

$\overline{S D}=\sqrt{S D_{x}^{2}+S D_{y}^{2}+S D_{z}^{2}}$.

In Fig. 5 one can see the evolution of the line topology with increasing $h_{0}$. The most clear trend is related to the diagonal lines (or stripes) which are fairly clear for lower $h_{0}$ and fairly smeared for higher $h_{0}$ signaling transition to a less deterministic motion. In recurrence analysis it is also important to analyze vertical structures. These structures are used to extract information about the transition from laminar to turbulent motion. Note that much shorter vertical lines (comparing to diagonal ones) are also present in the recurrence 

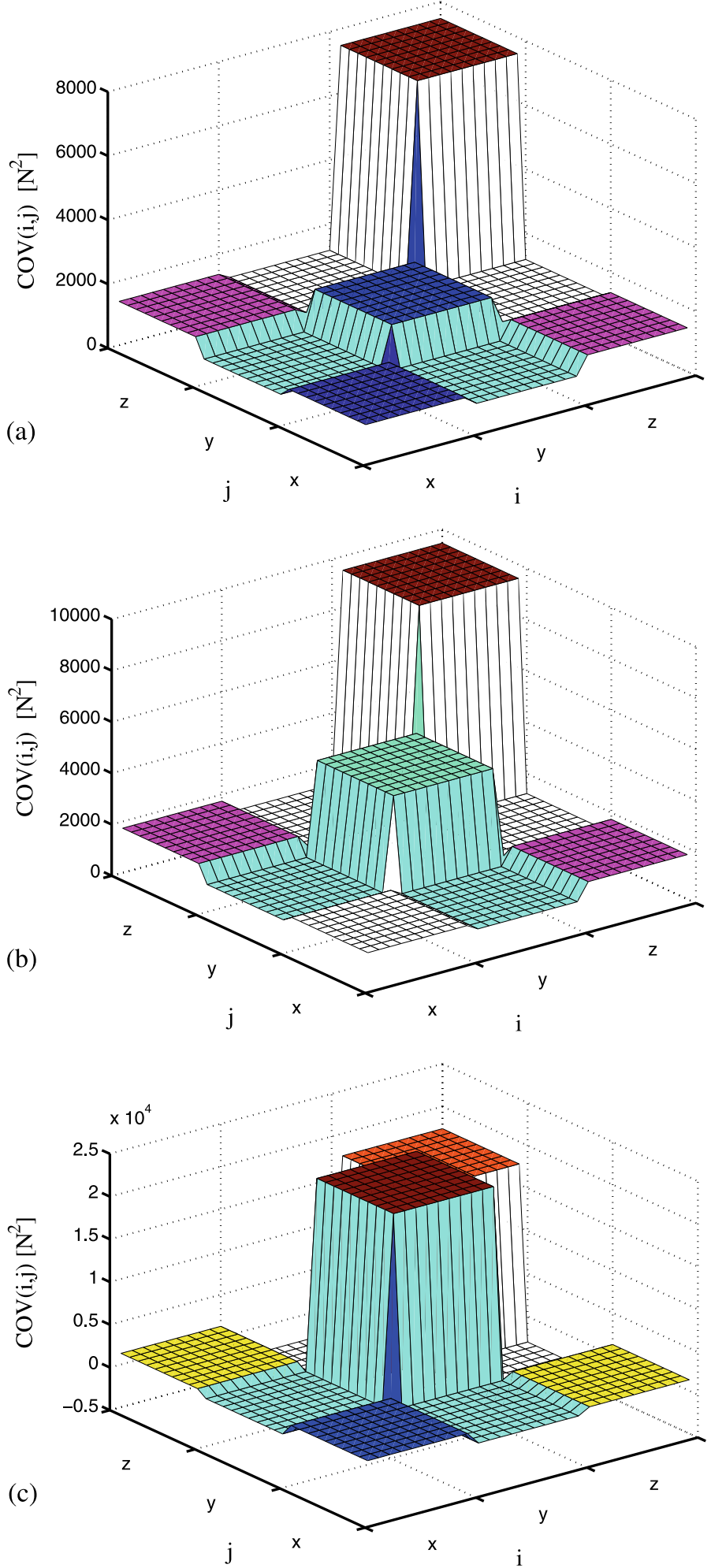

Fig. 4 The covariance matrix $\operatorname{COV}(i, j)$, where $i, j=x, y, z$ for $F_{x}, F_{y}$, and $F_{z}$ force components, respectively 

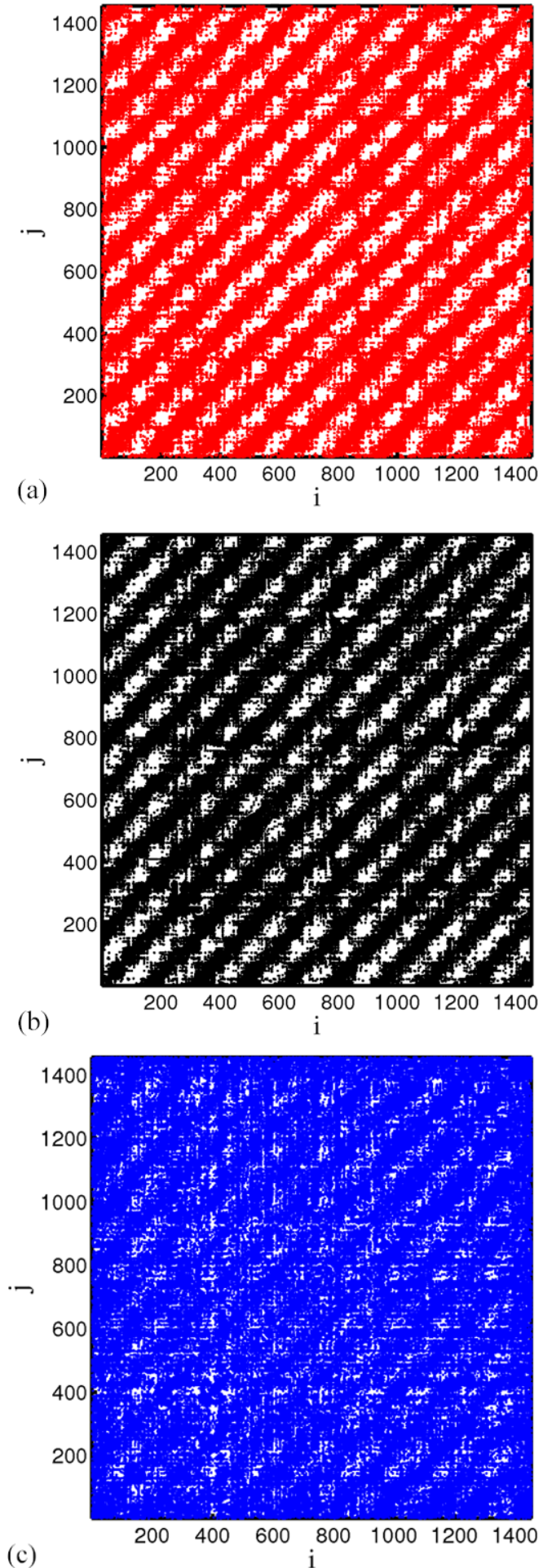

Fig. 5 Recurrence plots for different cutting depths $h_{0}=1.00$, 1.75 , and $2.30 \mathrm{~mm}$ for (a), (b), and (c), respectively. The threshold $\epsilon$ has been chosen as $S D$. Statistical and recurrence parameters are summarized in Table 2
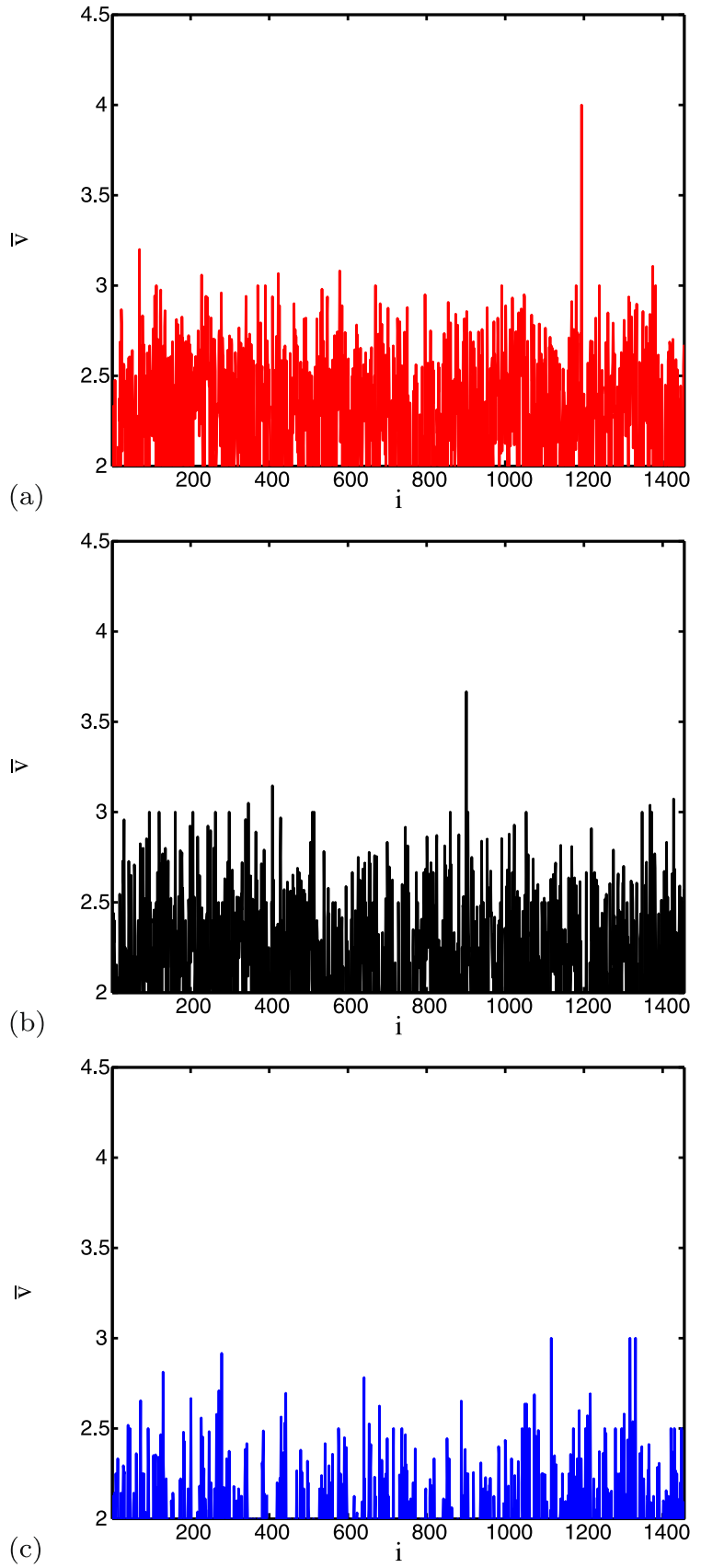

Fig. 6 Average vertical line $\bar{v}$ versus sampling index $i$ for different cutting depths $h_{0}=1.00,1.75$, and $2.30 \mathrm{~mm}$ for (a), (b), and $(\mathbf{c})$, respectively. The threshold $\epsilon$ has been chosen as in Fig. 5 with the cutoff $v>v_{\min }=2$ to eliminate singular points

plots (Fig. 5). Basing on these plots (Fig. 5) we show the distribution of the length $v$ of vertical lines averages (for $v>v_{\min }=2$ ) versus sampling points $i$. In Fig. 6 one can see that average lengths $\bar{v}$ have 
higher values of smaller $h_{0}$. Moreover one can see $\bar{v}$ riches the maxima for some selected $i$. Such maxima (as $i \approx 1200$ for Fig. 6(a)) and (as $i \approx 900$ for Fig. 6(b)) corresponds to the longer line appearance in Figs. 5(a)-(b). On the other hand, in case of the higher value of $h_{0}\left(h_{0}=2.30 \mathrm{~mm}\right.$, the average line lengths are the shortest among the examined cases. Note that their sampling time distribution $(\bar{v}$ against $i$ in Fig. 6(c)) is characterized by larger fluctuations comparing to Fig. 6(a)-(b). The fairly smaller values of $\bar{v}$ and also their wider distribution could be explained as a consequence of the presence of singular points. An increasing number of singular points in the vertical structure (for $v=1$ ) indicates that the system is more turbulent [32]. The further differences between the examined cases are better expressed by the statistical analysis of recurrence plots discussed below and summarized in Table 2.

Motivated by the fastest increase of variations (Table 1) and small off-diagonal covariance matrix elements (Fig. 4, (2)) we analyzed the recurrences for the singular $F_{y}$ force element, separately. The results for increasing $h_{0}$ are plotted in Fig. 7(a)-(c). The threshold $\epsilon$ was chosen as $0.4 S D_{y}$. In both sets of figures Figs. 5(a)-(c) and 7(a)-(c), after careful inspection, one can observe that the patterns of vertical lines responsible for the periodic system response are progressively destroyed by the growing cutting depth $h_{0}$.

This is understandable, because the change in $F_{y}$ is dominant in the system. This evolution is more transparent for Figs. 7(a)-(c). Note also the characteristic vertical lines structure (note, vertical lines are much shorter then diagonal ones) in Figs. 5(b) and 7(b). these effect implies the intermittency mechanism. Note that Fig. 5(c) and especially Fig. 7(c) show more homogeneous distribution of recurrence points implying more random behavior of the examined system. Note that recurrence plots in Figs. 5 and 7 include also singular points excluded in Fig. 6 and RQA.

To find out more about system dynamics we used the Recurrence Quantification Approximation (RQA) [32-36]. The fraction of recurrences with respect to all off-diagonal matrix elements is called Recurrence Rate $(R R)$

$R R=\frac{1}{N^{2}} \sum_{i, j}^{N} R_{i j}$.

Furthermore, the RQA can be used to identify diagonal and vertical lines through their lengths $l, v$ for

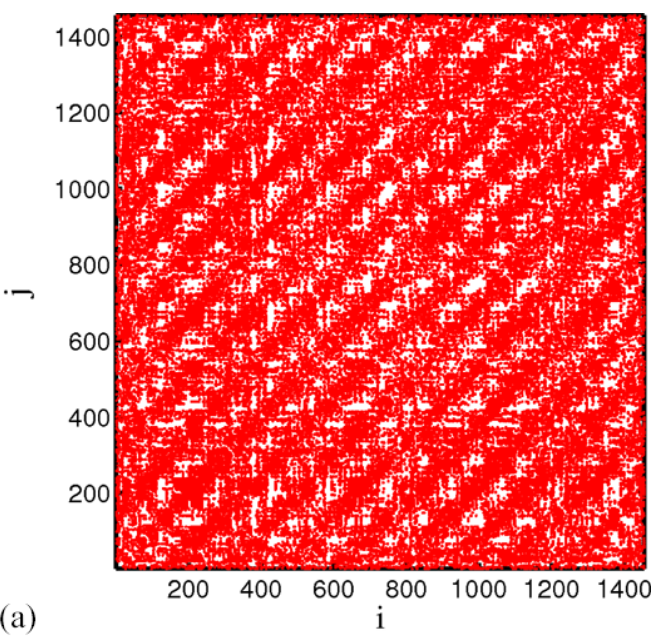

(a)

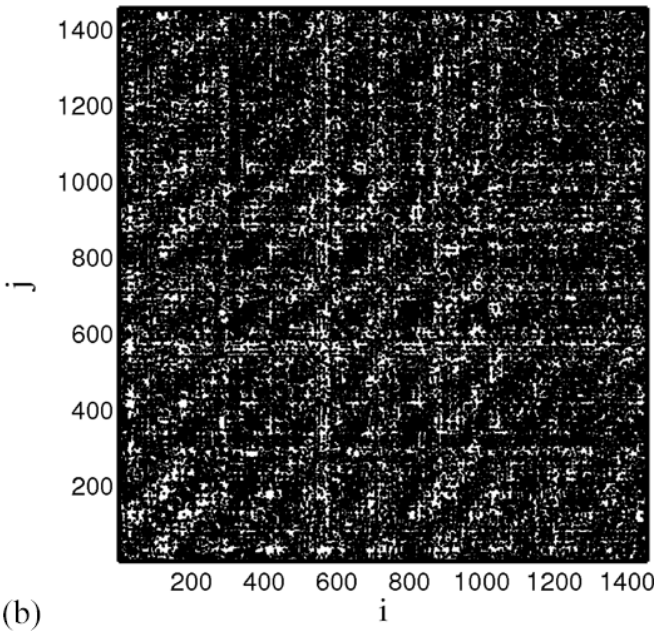

(b)

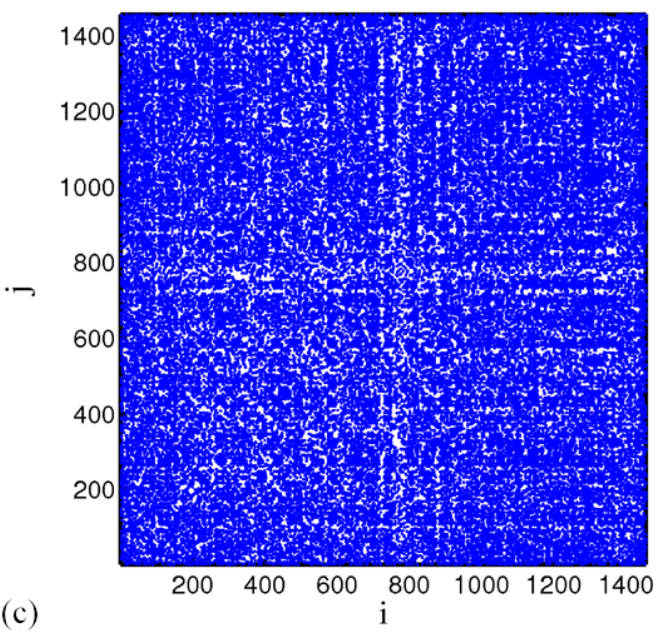

Fig. 7 Recurrence plots for the $y$ force component and for different cutting depths $h_{0}=1.00,1.75$, and $2.30 \mathrm{~mm}$ for (a), (b), and (c), respectively. The threshold $\epsilon$ has been chosen as $0.4 S D_{y}$. Statistical and recurrences parameters are summarized in Tables 1 and 2 
Table 2 Summary of RQA analysis. Different parameters for all force components $x y z$ (denoted by $X(1)$ ) calculated for $\epsilon=1.0 \overline{S D}_{k}(k=1,2,3)$. Different parameters for a single force component $z$ (denoted by $X(2)$ ) calculated for $\epsilon=0.4 S D_{k}(k=$ $1,2,3)$. $k$ corresponds to different cutting depths $h_{0}=1.00$, 1.75 , and $2.30 \mathrm{~mm}$

\begin{tabular}{lllllllll}
\hline $\begin{array}{l}\text { Cutting depth } \\
h_{0}[\mathrm{~mm}]\end{array}$ & \multirow{2}{*}{$R R(1)$} & $\begin{array}{l}D E T(1) / \\
R R(1)\end{array}$ & $\begin{array}{l}L A M(1) / \\
D E T(1)\end{array}$ & $\begin{array}{l}L_{E N T R}(1), \\
\hat{L}_{E N T R}(1)\end{array}$ & $R R(2)$ & $\begin{array}{l}\text { DET(2)/ } \\
R R(2)\end{array}$ & $\begin{array}{l}\text { LAM(2)/ } \\
D E T(2)\end{array}$ & $\begin{array}{l}L_{E N T R}(2), \\
\hat{L}_{E N T R}(2)\end{array}$ \\
\hline 1.00 & 0.0870 & 3.528 & 1.083 & $0.729,0.781$ & 0.0635 & 1.954 & 1.690 & $0.263,0.267$ \\
1.75 & 0.0771 & 3.652 & 1.057 & $0.650,0.674$ & 0.0414 & 2.073 & 1.442 & $0.257,0.285$ \\
2.30 & 0.0695 & 3.671 & 0.625 & $0.635,0.813$ & 0.0362 & 3.546 & 0.4569 & $0.298,0.344$ \\
\hline
\end{tabular}

diagonal and vertical lines, respectively. In its frame RQA provides us with the probability $p(l)$ or $p(v)$ of line distribution according to their lengths $l$ or $v$.

Practically, they are calculated by the following expression [36]:

$p(x)=\frac{P^{\epsilon}(x)}{\sum_{x=x_{\text {min }}}^{N} P^{\epsilon}(x)}$,

where $x=l$ or $v$ depending on diagonal or vertical structures in the specific recurrence diagram. $P^{\epsilon}(x)$ denotes the unnormalized probability for a given threshold value $\epsilon$. In this way Shannon information entropy $\left(L_{E N T R}\right)$ can be defined for diagonal line collections

$L_{E N T R}=-\sum_{l=l_{\min }}^{N} p(l) \ln p(l)$,

Other properties, such as determinism $D E T$ and laminarity $L A M$ are also basing on probabilities $P^{\epsilon}(x)$ :

$D E T=\frac{\sum_{l=l_{\min }}^{N} l P^{\epsilon}(l)}{\sum_{i, j=1}^{N} l P^{\epsilon}(l)}$,

$L A M=\frac{\sum_{v=v_{\min }}^{N} v P^{\epsilon}(v)}{\sum_{v=1}^{N} v P^{\epsilon}(v)}$,

where $l_{\min }$ and $v_{\min }$ denotes minimal values which should be chosen for a specific dynamical system. In our calculations we have assumed $l_{\text {min }}=v_{\text {min }}=2$.

Determinism DET is the measure of the predictability of the examined time series and gives the ratio of recurrent points formed in diagonals to all recurrent points. Note in a periodic system all points would be included in the lines. On the other hand laminarity $L A M$ is a similar measure which corresponds to points formed in vertical lines. This measure indicates the dynamics behind sampling point changes.
For small point to point changes (by $i \rightarrow i+1$ or $j \rightarrow j+1)$ the consecutive points $\left(R_{i j}=1\right)$ form a vertical line.

Note that the definition of frequency $p(l)$ and consequently the probability $P^{\epsilon}$ calculation equation (7) does not consider the information about shorter lengths $l_{2}$ while it is embedded in longer one $l_{1}\left(l_{1}>l_{1}\right)$. After renormalization of $p(l)$ with respect of these possibilities we obtain the new frequencies $p^{*}(l)$ which can be used to define the Renyi entropy $\hat{L}_{\text {ENTR }}$ :

$\hat{L}_{E N T R}=-\sum_{l=l_{\min }}^{N} p^{*}(l) \ln p^{*}(l)$,

The results of RQA analysis are presented in Table 2 . Note that taking into account that for the same epsilon there are different number of recurrences (colored points on RP-Figs. 5 and 7) we normalized DET by $R R$. Similarly locking for the laminarity we expressed a ratio $L A M / D E T$ which can tell us about the formation of vertical structures with respect to fraction of points laing in diagonal formations (chaoticity or turbulence is the opposite of laminarity).

As expected after statistical (Table 1) and qualitative analysis by RP (see Figs. 5 and 7) the ratio $L A M / D E T$ indicates the monotonic change of the nonregular dynamics contributions. Examining the other RQA parameters we report that $R R$ is also changing monotonically. $R R$ is the topological measure of correlation of the time series [32] in terms of recurrences. It appears that for the same range of threshold $\epsilon$ (expressed in units of the corresponding square deviation) $R R$ is smaller for larger $h_{0}$. That means that the signal becomes less correlated. $D E T / R R$ shows the increasing tendencies with growing $h_{0}$ while $L A M / D E T$ shows decreasing ones. Evidently, it is related to in- 
creasing of randomnesses and non-laminarities, respectively.

As far as the entropy off diagonal lines is concerned the results depend of the probability definition $\left(p\right.$ or $\left.p^{*}\right)$. The Renyi entropy $\left(\hat{L}_{E N T R}\right)$ in both cases, the full force $F_{x}, F_{y}, F_{z}$ and single component $F_{y}$ representations, exhibit its maximum value for the largest cutting depth $h_{0}$. The monotonic increase of $\hat{L}_{E N T R}(2)$ signals the growing unpredictability (larger noise or/and chaos contribution). Note that $\hat{L}_{E N T R}(2)$ is ambiguous as the $F_{y}$ component is weakly correlated with the rest of force components. It is clear that for the small $h_{0}$ vibrations are more collective, exhibiting the same period in all force components, while for the larger $h_{0}$ new physical effects have been developed. This could be effect of nonlinear effect of friction. The increasing tangential component $F_{z}$ lead to the stronger friction force between the tool and the chip (part of $F_{y}$ ). These could lead to the intermittency of the stick-and-slip limit cycle [37, 38].

\section{Conclusions}

This work presents experimental investigations of cutting process in which the stainless steel was cut. During the experiment three component cutting forces are measured as a function of the assumed cutting depth $h_{0}$.

As expected, the increasing cutting depth leads to higher values of all cutting forces components. Following this trend, the fluctuations of force values are also increasing showing, however, different distributions for different force components. These were a subject of our interest. The obtained signals were analyzed using statistical and recurrence methods. We claim for larger cutting force component the frictional stick-and-slip mechanism of self-sustained vibrations $[12,37,38]$ can be more important than the regenerative effect $[5,13,26]$. However the main period of vibrations is caused by the rotational motion of an unbalanced workpiece shaft.

The most interesting contribution was found in the $y$ force component. Its local non-monotonous change of kurtosis signals an interesting change in the system dynamic response. The recurrence plots are clearly indicating that the response is less periodic (or more chaotic) for larger cutting depth $h_{0}$. The obtained results can be used to identify types of vibrations and select the most proper cutting parameters including the cutting depth $h_{0}$ and the angular speed of the workpiece.

Note that the recurrence plots and recurrence quantification (by the $R R$ parameter) are tools suitable to analyze fairly short time series [29, 31, 32]. To draw more conclusions we will study the fluctuations more systematically by including intermediate cutting depths, various angular speeds, and consider longer time series to apply additional dynamical tests such as Fourier and wavelet analysis.

Acknowledgements The financial support of Structural Funds in the Operational Programme-Innovative Economy (IE OP) financed from the European Regional Development FundProject "Modern material technologies in aerospace industry", No. POIG.01.01.02-00-015/08-00 is gratefully acknowledged.

Open Access This article is distributed under the terms of the Creative Commons Attribution Noncommercial License which permits any noncommercial use, distribution, and reproduction in any medium, provided the original author(s) and source are credited.

\section{References}

1. Taylor F (1907) On the art of cutting metals. Trans ASME 28:31-350

2. Arnold RN (1946) The mechanism of tool vibration in the cutting of steel. Proc Inst Mech Eng 154:261-284

3. Tobias SA, Fishwick W (1958) A theory of regenerative machine Tool chatter. The Engineer, London

4. Stepan G (2001) Modelling nonlinear regenerative effects in metal cutting. Philos Trans R Soc Lond A 359:739-757

5. Fofana MS (2003) Delay dynamical systems and applications to nonlinear machine-tool chatter. Chaos, Solitons \& Fractals 12:731-747

6. Wang XS, Hu J, Gao JB (2006) Nonlinear dynamics of regenerative cutting processes-Comparison of two models. Chaos, Solitons \& Fractals 29:1219-1228

7. Tlusty J, Polacek M (1963) The stability of machine tool against self-excited vibrations in machining. ASME Int Res Prod Eng 465-474

8. Merrit HE (1965) Theory of self-excited machine-tool chatter. ASME J Eng Ind 87:447-454

9. Pratt JR, Nayfeh AH (2001) Chatter control and stability analysis of a cantilever boring bar under regenerative cutting conditions. Philos Trans R Soc Lond A 359:759-792

10. Wu DW, Liu CR (1985) An analytical model of cutting dynamics. Part 1: Model building. ASME J Eng Ind 107: 107-111

11. Wu DW, Liu CR (1985) An analytical model of cutting dynamics. Part 2: Verification. ASME J Eng Ind 107:112-118

12. Grabec I (1988) Chaotic dynamics of the cutting process. Int J Mach Tools Manuf 28:19-32

13. Stépán G, Szalai R, Insperger T (2003) Nonlinear dynamics of high-speed milling subjected to regenerative effect. In: Radons G, Neugebauer R (eds) Nonlinear dynamics of production systems. Wiley, New York 
14. Vela-Martínez L, Jáuregui-Correa JC, González-Brambila OM, Herrera-Ruiz G, Lozano-Guzmán A (2009) Instability conditions due to structural nonlinearities in regenerative chatter. Nonlinear Dyn 56:415-427

15. Vela-Martínez L, Jáuregui-Correa JC, Álvarez-Ramirez J (2009) Characterization of machining chattering dynamics: An R/S scaling analysis approach. Int J Mach Tools Manuf 49:832-842

16. IN Tansel, Erkal C, Keramidas T (1992) The chaotic characteristics of three-dimensional cutting. Int $\mathrm{J}$ Mach Tools Manuf 32:811-827

17. Gradisek J, Govekar E, Grabec I (1996) A chaotic cutting process and determining optimal cutting parameter values using neural networks. Int J Mach Tools Manuf 36: 1161-1172

18. Wiercigroch M (1997) Chaotic vibration of a simple model of the machine tool-cutting process system. J Vib Acoust 119:468-475

19. Wiercigroch M, Cheng A (1997) Chaotic and stochastic dynamics of orthogonal metal cutting. Chaos Solitons Fractals 8:715-726

20. Gradisek J, Govekar E, Grabec I (1998) Time series analysis in metal cutting: chatter versus chatter-free cutting. Mech Syst Signal Process 12:839-854

21. Gradisek J, Govekar E, Grabec I (1998) Using coarsegrained entropy rate to detect chatter in cutting. J Sound Vib 214:941-952

22. Marghitu DB, Ciocirlan BO, Craciunoiu N (2001) Dynamics in orthogonal turning process. Chaos Solitons Fractals $12: 2343-2352$

23. Litak G (2002) Chaotic vibrations in a regenerative cutting process. Chaos Solitons Fractals 13:1531-1535

24. Gradisek J, Grabec I, Sigert S, Friedrich R (2002) Stochastic dynamics of metal cutting: bifurcation phenomena in turning. Mech Syst Signal Process 16:831-840

25. Litak G, Rusinek R, Teter A (2004) Nonlinear analysis of experimental time series of a straight turning process. Meccanica 39:105-112

26. Altintas Y (2000) Manufacturing automation: Metal cutting mechanics, machine tool vibrations, and $\mathrm{CNC}$ design. Cambridge University Press, Cambridge
27. Takens F (1981) Detecting strange attractors in turbulence. In: Rand DA, Young L-S (eds) Dynamical systems and turbulence. Lecture notes in mathematics, vol 898. Springer, Berlin, pp 366-381

28. Kantz H, Schreiber T (1997) Non-linear time series analysis. Cambridge University Press, Cambridge

29. Litak G, Wiercigroch M, Horton BW, Xu X (2010) Transient chaotic behavior versus periodic motion of a pendulum by recurrence plots. Z Angew Math Mech 90:33-41

30. Litak G, Sawicki JT, Kasperek R (2009) Cracked rotor detection by recurrence plots. Nondestruct Test Eval 24: 347-351

31. Friswell MI, Litak G, Sawicki JT (2010) Crack identification in rotating machines with active bearings. In: Proc of ISMA 2010, Leuven, Belgium, 20-22 September, pp 2843-2855

32. Marwan N, Romano MC, Thiel M, Kurths J, (2007) Recurrence plots for the analysis of complex systems. Phys Rep 438:237-329

33. Eckmann J-P, Kamphorst SO, Ruelle D (1987) Recurrence plots of dynamical systems. Europhys Lett 5:973-977

34. Webber CL, Zbilut JP (1994) Dynamical assessment of physiological systems and states using recurrence plot strategies. J Appl Phys 76:965-973

35. Marwan N (2003) Encounters with neighbours: current development of concepts based on recurrence plots and their applications. $\mathrm{PhD}$ thesis, Universität Potsdam, Potsdam

36. Marwan N (2010) Cross recurrence plot toolbox. http:// www.agnld.uni-potsdam.de/marwan/toolbox/ (Accessed 25 May 2010)

37. Wiercigroch M, Krivtsov AM (2001) Frictional chatter in orthogonal metal cutting. Philos Trans R Soc Lond A 359:713-738

38. Warminski J, Litak G, Cartmell MP, Khanin R, Wiercigroch M (2003) Approximate analytical solutions for primary chatter in the nonlinear metal cutting model. J Sound Vib 259:917-933 